\title{
Editorial
}

\section{More about genetically modified tumour vaccines}

The majority of gene therapy clinical trials are related to cancer. In turn, most human cancer gene therapy protocols are based on immunotherapy strategies, the genetically modified tumour vaccines (GMTV) being the most frequently applied. Such a status is most probably due to the historical background of cancer immunotherapy, data gathered in melanoma vaccine trials and current gene transfer technology. Moreover, immunology of some immunogenic tumours, such as malignant melanoma, is better understood. It is likely that GMTV approaches will be replaced with time by novel gene therapy strategies, but now serve as a tool for understanding some of the pathomechanisms of human cancer and provide potential for treatment. Since the malignant melanoma is the most frequent target for GMTV approaches, we will focus on this malignancy in the editorial.

It is becoming generally accepted that for elicitation of effective specific anticancer immune response $\mathrm{CD}^{+}$or $\mathrm{CD}^{+}$lymphocytes require at least three synergistic signals: (1) presentation of tumour rejection antigens (TRA) to $\mathrm{T}$ cell receptor (TCR) on $\mathrm{CD}^{+}$by tumour cells in the context of HLA class I molecules or on $\mathrm{CD}^{+}$by professional antigen-presenting cells (APC) in the context of HLA class II; (2) non-antigen specific accessory/costimulatory signals, eg interaction of CD80 and CD86 on APC with CD28 on T cells; (3) proliferative signal provided by cytokines or growth factors in order to recruit and propagate tumour-reactive lymphocytes. ${ }^{1}$ Abnormalities in providing or receiving these signals, eg due to the loss or down-regulation of HLA I (as well as HLA II), defective machinery involved in antigen processing or impaired signal transduction in $\mathrm{T}$ cells may lead to the tumour escape from the immunological control. Moreover, other factors such as transforming growth factor $\beta$ (TGF- $\beta$ ) or IL-10 secreted by the tumour, or Fas expressed on the tumour cells may inhibit tumour elimination. Both induction and effector phases of the antitumour immunological response might be affected. Although malignant melanoma cells display an antigenic profile which in part is similar to natural APC, they are poorly immunogenic for $\mathrm{T}$ cells. Accordingly, the principle of GMTV is to modify tumour cells by insertion of genes encoding various factors to enhance their immunogenicity in order to induce (initiate) specific antitumour responses.

Three major questions arise when designing GMTV: (1) Which gene should be used for modification of the target cell? (2) Which cell would form the basis of the vaccine?
(3) Which gene delivery system should be applied? Irradiation of tumour cells with sterilizing doses became a routine step in the GMTV preparation process for several reasons: safety, increase of the HLA I and II expression and increase of production of the cytokine encoded by the inserted gene. Until now cytokine and growth factor genes were most frequently used for tumour cell modification. A large number of cytokines such as IL-2, IL-4, IL-7, IL-12, interferons (IFN) or GMCSF are currently being tested in clinical trials. Each cytokine displays unique biological activities in the system, eg IFN- $\gamma$ may increase expression of HLA I and II molecules, TRA and costimulatory molecules, IL-2 enables expansion of activated T cells, while GM-CSF activates APC. In order to increase efficiency of GMTV some activities are being combined, thus cells are being modified by a combination of genes, eg IL-2 and IFN-gamma, IL-2/GM-CSF, IL-2/IL-7, IL-7/IL-12/GM-CSF.

Original GMTV design was based on modification of autologous tumour cells which demonstrated TRA restricted largely or uniquely to each individual and the same HLA context. However, from the practical point of view production of the autologous GMTV is time-consuming, dependent on the quantity of available tumour, and frequently not possible due to the tumour inaccessibility or problems with propagating cells in vitro. As an alternative to autologous cells, allogeneic cell lines which are HLA matched and sharing at least some TRAs with the patient's tumour can be used. Finally, so-called mixed GMTV comprising of unmodified autologous tumour cells and autologous fibroblasts modified to secrete cytokines were created.

Depending on the cell type forming the basis of GMTV, an appropriate gene delivery system should be chosen. In the case of autologous cells, most recommended would currently be the adenoviral vector system which is characterized by its ability to transduce non-dividing cells with high efficiency without the necessity for cell selection. Virus particles also serve as additional adjuvant due to the presence of highly immunogenic adenoviral proteins. Although inserted genes do not integrate into the genome of the target cells, protein is produced in sufficient amounts for at least a couple of weeks. In turn, for transduction of allogeneic cells the choice would be a retroviral vector system characterized by its ability to stably integrate the desired gene into the genome of the dividing target cell. Tissue-specific or highly efficient promoters can be used.

We have developed a novel GMTV approach for human melanoma immunotherapy in terms of using stimulatory molecules, admixture of autologous and 
modified allogeneic tumour cells and gene delivery systems. ${ }^{2}$ The novel stimulatory principle displaying unique biological activity comprises a complex of two proteins, IL-6 and its agonistic soluble $\alpha$ receptor subunit (sIL-6R). It activates cells both possessing and lacking the ligand binding IL-6 receptor $\alpha$. Cells which do not express IL-6 receptor $\alpha$ in the absence of the sIL-6R are unable to respond to IL-6.

In a tumour rejection model using $\mathrm{B} 78 \mathrm{H} 1$ murine melanoma cell line, showing no $\mathrm{H}-2^{\mathrm{b}}$ expression, we have demonstrated that sIL-6R significantly enhanced the IL6 effect on the inhibition of melanoma growth and metastases formation. Immunization of mice with $\mathrm{B} 78 \mathrm{H} 1$ cells transfected with IL-6 and sIL-6R genes stimulated long-lasting anti-melanoma immunity, while IL-6 alone had only a marginal effect. IL-6 (via CD4 $4^{+}$) and IL-6/sIL$6 \mathrm{R}$ complex (via $\mathrm{CD}^{+}$) have shown diverse mechanisms of activation of the induction phase of the anti-melanoma immune response. However, the effector phase was similar $\left(\mathrm{CD}^{+}\right)$, but much more intense in the case of induction by IL-6/sIL-6R.,

Two genes encoding IL-6 and sIL-6R were transduced into a human melanoma cell line positive for HLAA1/A2, as well as MAGE 1/3, MART-1 and tyrosinase using original double copy dicistronic vector containing a CMV-IE promoter. ${ }^{5}$ IL-6 and sIL-6R were secreted in quantities of 300 and $100 \mathrm{ng} / \mathrm{ml} / 10^{6}$ per $24 \mathrm{~h}$, respectively. These cells were admixed with isolated autologous cells (previously frozen in aliquots without propagation in culture), irradiated and injected into patients. ${ }^{6}$ At the site of injection of the majority of patients, vigorous DTH reaction was seen. Biopsy demonstrated heavy infiltrates of both $\mathrm{CD}^{+}$and $\mathrm{CD}^{+}\left(\mathrm{DR}^{+}\right) \mathrm{T}$ cells. Distal skin metastases, which macroscopically demonstrated typical signs of inflammatory reaction in the majority of patients, also showed heavy infiltrates of $\mathrm{CD}^{+}, \mathrm{CD}^{+}\left(\mathrm{DR}^{+}\right) \mathrm{T}$ cells. In one patient who did not show a DTH reaction, parallel administration of GMTV lacking autologous cells caused a very rigid DTH response.

Our results indicate that the IL-6/sIL-6R complex has potential to activate a broader spectrum of cells involved in the induction of specific anti-melanoma response than other factors. Moreover, preparation of GMTV according to our protocol is easy, low cost and fast. In the event of inaccessibility to autologous cells allogeneic GMTV might be applied. Finally, autologous tumour cells may inhibit not only the effector phase but also the induction phase of immune response which should be considered when designing a clinical protocol employing GMTV based on autologous cells.

\section{Acknowledgements}

This work was supported by the State Committee for Scientific Research (Warsaw, Poland) and the Deutsche Forschungsgemeinschaft (Bonn, Germany).

A Mackiewicz ${ }^{1}$ and S Rose-John ${ }^{2}$

${ }^{1}$ Department of Cancer Immunology University School of Medical Sciences Garbary St 1561866 Poznan, Poland; and ${ }^{2} I$ Medical Clinic, Faculty of Medicine J Gutenberg University of Mainz, Germany

\section{References}

1 Bubenik J. Gene transfer for immunotherapy of cancer. Gene Therapy 1996; 3: 944-945.

2 Mackiewicz A, Rose-John S. Anticancer vaccine comprising of IL-6/sIL-6 receptor transfected cells. International patent PCT/PL 96/00010.

3 Mackiewicz A et al. Soluble interleukin-6 receptor is biologically active in vivo. Cytokine 1995; 7: 142-149.

4 Mackiewicz A et al. Interleukin-6-type cytokines and their receptors for gene therapy of melanoma. Ann NY Acad Sci 1995; 762. 361-374.

5 Wiznerowicz M, Fong AC, Mackiewicz A, Hawley RC. Development of dicistronic double copy retroviral vectors for human gene therapy. Gene Therapy 1997; 4: 1061-1068.

6 Mackiewicz A et al. Gene therapy of human melanoma. Immunization of patients with autologous tumor cells admixed with allogeneic melanoma cells secreting interleukin 6 and soluble interleukin 6 receptor. Hum Gene Ther 1995; 6: 805-811. 